\title{
Analysis of the nodal beam isolation system based on application of helical spring
}

Received: 13 April 2021 / Revised: 5 July 2021 / Accepted: 12 July 2021 / Published online: 9 August 2021 (C) The Author(s) 2021

\begin{abstract}
A nodal beam isolation system allows the transmission of vibration from the source to an isolated element to be limited using nodal points on the elastic beam connecting them. These points are selected in such a way that their position during vibration is constant. The application of a helical spring as an elastic beam reduces the dimensions of the system and increases its applications. An effective computational model of the nodal beam isolation system based on a helical spring application as an elastic beam is presented in the paper. The model allows the position of nodal points to be determined for a given excitation frequency. It also allows the influence of system parameters on spring vibration amplitudes and static stiffness of the connection between the source and isolated element to be analysed. The analysis makes it possible to formulate conclusions facilitating the designer to select the proper system parameters for the given operating conditions. The results of numerical and experimental tests exhibit high compliance with the results of the presented model.
\end{abstract}

\section{Introduction}

The nodal beam vibration isolation system consists of a vibrating object connected to an isolated structure by means of an elastic beam. Its axis is perpendicular to the direction of vibrations, as shown in Fig. 1. The external force of amplitude $P$ excites vibrations with such a frequency $\omega$ that two nodal points appear symmetrically along the beam axis at which the vibration displacement is zero.

This method can be applied to isolate systems vibrating with a constant frequency. The most well-known application of this method is Bell's NodaMatic nodalised beam concept [1], which minimises vibration transfer from the rotorhead to the fuselage of a helicopter [2]. In constant frequency vibration, the nodal beam isolation method exhibits significant advantages compared to conventional passive vibration isolation systems. The main advantage of this method is that no vibration is transmitted to an isolated object for a perfectly tuned system. The second important advantage is the relatively high static stiffness of the connection between the vibration source and the isolated object. Thanks to these advantages, this method can be applied to control handle vibration in machines such as jackhammers, rammers, plate compactors, hand tractors, or grass trimmers [3]. Operators of such machines experience high levels of vibration in the hand and arm [4-8], which can lead to health problems [9-12] called hand-arm vibration syndrome (HAVS). The frequency of vibrations generated in such machines varies from several up to several dozen $\mathrm{Hz}$, which depends mostly on their engine/motor rotational speed. Utilizing the nodal beam method for such a range of excitation frequency requires the application of

K. Michalczyk $(\varangle) \cdot$ W. Sikora

Faculty of Mechanical Engineering and Robotics, AGH University of Science and Technology, A. Mickiewicza Ave. 30, 30-059 Cracow, Poland e-mail:kmichal@agh.edu.pl

W. Sikora

e-mail:wosikora@agh.edu.pl 


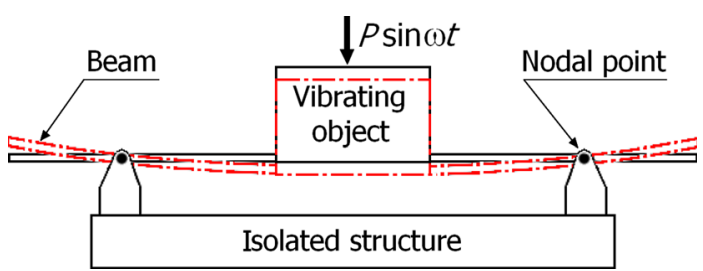

Fig. 1 Nodal beam vibration isolation system scheme

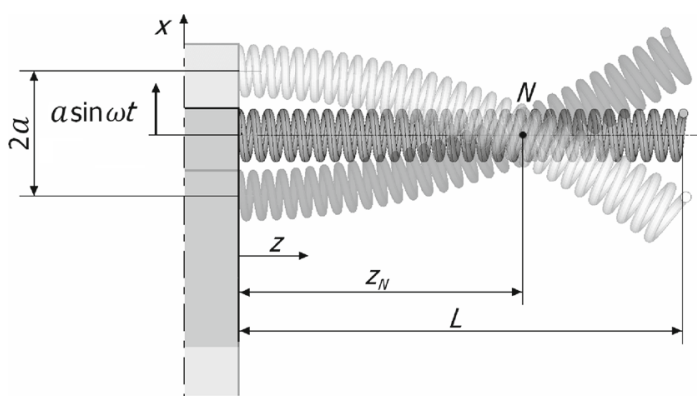

Fig. 2 Scheme of the described model. Due to its symmetry, only half of it is shown

steel leaf springs of considerable length and, consequently, large mass to obtain suitable elastic and inertial parameters of the system.

The inconvenience caused by the dimensions of a leaf spring can be overcome by replacing it with a steel helical spring. Such a solution was described in [13], where the authors employed a helical spring in experimental research. However, they used the Euler beam model for analytical calculations. While this is acceptable for slender solid prismatic shapes, applying such a model for a spring can lead to incorrect results.

The elastic and inertial properties of helical springs made of selected material can be tuned in a wide range, while maintaining appropriate external dimensions. This is so because these properties are a function of four geometric parameters: the wire diameter $d$, spring index $C=D / d$ (where $D$ is the nominal spring diameter), lead angle $\delta$ and number of active coils $n$. The selection of spring parameters should ensure, at the same time, a relatively high transverse stiffness of the connection between the vibrating object and the isolated structure, low maximum amplitudes of spring transverse vibrations in working conditions, and small dimensions of the system. In order to deal with this issue, it is necessary to develop an effective computing tool that will allow us to obtain the most favourable spring parameters for the given working conditions.

The aim of this study is the analysis of a nodal beam vibration isolation system based on a helical spring and creation of a model allowing parameters of the spring to be selected in order to meet given requirements.

\section{Model of the analysed system}

The model of the system based on the concept of a nodal beam, where the role of an elastic element is carried out by a helical spring, is shown in Fig. 2. The left end of the presented spring model illustrated in Fig. 2 is mounted in the fixture, which oscillates in the vertical direction with the angular frequency $\omega$ and amplitude $a$. Movement of the fixture causes transverse vibration of the spring in such a way that the $x$-displacement of the selected point $N$ on the spring axis measured in a stationary coordinate system is equal to zero at every instance of time.

The nodal point $N$ can therefore be used as a place for mounting the isolated object. The condition of the proper functioning of such a system is to ensure the free rotation of the spring axis at the point $N$ around the axis perpendicular to the plane in which vibrations occur.

A helical spring can be modelled as an equivalent Timoshenko beam. As was shown in [14] among others, this model gives results very close to the results presented in other works $[15,16]$, where the spring was modelled as a spatially curved rod. Thanks to its lower complexity, the equivalent beam model is simpler in application and therefore more efficient for the purpose of system analysis. 
Based on the concept of an equivalent beam, dynamic motion equations for excited transverse vibrations of a spring can be written as below [17]:

$$
\begin{gathered}
\beta\left(w^{\prime \prime}-\psi^{\prime}\right)-c_{1} \dot{w}-m_{u} \ddot{w}=-p(z, t), \\
\alpha \psi^{\prime \prime}+\beta\left(w^{\prime}-\psi\right)-c_{2} \dot{\psi}-m_{u} r_{g}^{2} \ddot{\psi}=0 .
\end{gathered}
$$

The coefficients $\alpha$ and $\beta$ are equivalent bending and shearing rigidities of the spring, respectively [18]:

$$
\alpha=\frac{2 E J \sin \delta}{2+\nu \cos ^{2} \delta} \beta=\frac{2 E J \sin \delta}{R^{2}\left(1+\nu \sin ^{2} \delta\right)},
$$

where $E J$ is the product of the Young's modulus and the area moment of inertia of the wire cross section, $v$ is the Poisson's ratio and $R$ is half of the spring diameter $D$. The coefficients $c_{1}$ and $c_{2}$ correspond to translational and rotational damping effects, respectively, $m_{u}$ is the spring mass per unit length, while $r_{g}$ represents the radius of gyration of the equivalent beam cross section. The angle $\psi$ is the angle of the spring axis deflection caused only by bending, $w$ denotes the spring axis transverse displacement resulting from bending and shearing and $p(z, t)$ denotes the continuous transverse load applied to the spring.

The effects associated with damping in the case of steel springs can be omitted as the damping of steel is negligibly small. Under this assumption, the separation of variables can be performed using the following substitutions: $w(z, t)=W(z) \sin \omega t, \psi(z, t)=\Psi(z) \sin \omega t$ and $p(z, t)=P(z) \sin \omega t$. After proper transformations, Eq. (1) can be rewritten in the form

$$
\begin{gathered}
W^{\prime \prime}-\Psi^{\prime}+\omega^{2} \frac{m_{u}}{\beta} W=\frac{-1}{\beta} P, \\
\psi^{\prime \prime}+\frac{\beta}{\alpha}\left(W^{\prime}-\Psi\right)+\omega^{2} \frac{m_{u} r_{g}^{2}}{\alpha} \Psi=0 .
\end{gathered}
$$

In the analysed case shown in Fig. 1, harmonic transverse oscillations of the clamped end of the spring correspond to the inertial excitation of constant amplitude- $P(z)=$ const. The relationship between the amplitude of transverse oscillations $a$ and the inertia load amplitude $P$ takes the form $P=m_{u} \omega^{2} a$. By introducing into Eq. (3) new dependent variables in a known way:

$$
\bar{W}=W+a, \bar{\Psi}=\Psi
$$

we obtain motion equations of the spring in an identical form as natural vibration equations:

$$
\begin{gathered}
\bar{W}^{\prime \prime}-\bar{\Psi}^{\prime}+\omega^{2} \frac{m_{u}}{\beta} \bar{W}=0, \\
\bar{\Psi}^{\prime \prime}+\frac{\beta}{\alpha} \bar{W}^{\prime}+\left(\omega^{2} \frac{m_{u} r_{g}^{2}}{\alpha}-\frac{\beta}{\alpha}\right) \bar{\Psi}=0 .
\end{gathered}
$$

It should be noted that, in the considered case of kinematically excited transverse vibrations, the variable $W$ describes transverse displacements in a local coordinate system connected with the left end of the spring (Fig. 1), while the variable $\bar{W}$ describes transverse displacements in a global, fixed coordinate system. After proper transformations of Eq. (5), two differential equations separately describing $\bar{W}$ and $\bar{\Psi}$ can be obtained. Their steady solution forms depend on the relation between the excitation frequency $\omega$ and the cut-off frequency $\omega_{b}$, which in turn depends on the material and geometrical spring parameters. The excitation frequency $\omega$ in the nodalised beam system should be higher than the first natural frequency $\omega_{I}$ and lower than the second natural frequency $\omega_{I I}$. As proved in one paper [14], the relationship between successive natural frequency values of transverse vibrations and the value of $\omega_{b}$ in the case of an uncompressed helical spring depends only on the spring slenderness value $\lambda=L / D$. It was shown in the quoted paper that, even in the case of springs with small slenderness values $(\lambda \approx 1.5)$, the two first natural frequencies of transverse vibrations under clamped-clamped boundary conditions have smaller values than the cut-off frequency. For higher slenderness values, this relation is also maintained. Natural frequencies of a clamped-free spring are lower than analogous frequencies of a clamped-clamped spring; thus in this study, it was assumed that the excitation frequency $\omega$ is lower than $\omega_{b}$. Under this assumption, the steady-state solutions of differential equations describing $\bar{W}$ and $\bar{\Psi}$ take the form

$$
\bar{\Psi}(z)=C_{1} \cosh k_{1} z+C_{2} \sinh k_{1} z+C_{3} \cos k_{2} z+C_{4} \sin k_{2} z
$$




$$
\bar{W}(z)=A_{1}\left(C_{1} \sinh k_{1} z+C_{2} \cosh k_{1} z\right)+A_{2}\left(C_{3} \sin k_{2} z-C_{4} \cos k_{2} z\right),
$$

where

$$
k_{1,2}=\sqrt{\mp\left(\frac{1}{\beta}+\frac{r_{g}^{2}}{\alpha}\right) \frac{m_{u} \omega^{2}}{2}+\sqrt{\frac{\left(m_{u}\left(\beta r_{g}^{2}-\alpha\right)^{2} \omega^{2}+4 \alpha \beta^{2}\right) \omega^{2} m_{u}}{m_{u} \omega^{2}}, \quad A_{2}=r_{g}^{2} k_{2}-\frac{\alpha k_{2}^{3}}{m_{u} \omega^{2}},}} .
$$

The boundary conditions in the analysed case take the form:

$$
1:\left.\bar{\Psi}\right|_{z=0}=0, \quad 2:\left.\bar{W}\right|_{z=0}=a, \quad 3:\left.\bar{\Psi}^{\prime}\right|_{z=L}=0,4:\left.\left(\bar{W}^{\prime}-\bar{\Psi}\right)\right|_{z=L}=0
$$

where $\bar{W}^{\prime}-\bar{\Psi}$ represents the amplitude of the angle of the spring axis deflection caused only by shearing:

$$
\bar{W}^{\prime}(z)-\bar{\Psi}(z)=A_{3}\left(C_{1} \cosh k_{1} z+C_{2} \sinh k_{1} z\right)+A_{4}\left(C_{3} \cos k_{2} z+C_{4} \sin k_{2} z\right),
$$

where

$$
A_{3}=\frac{-\alpha k_{1}^{2}-m_{u} r_{g}^{2} \omega^{2}}{\beta}, \quad A_{4}=\frac{\alpha k_{2}^{2}-m_{u} r_{g}^{2} \omega^{2}}{\beta} .
$$

Using the boundary conditions (8), the coefficients $C_{1,2,3,4}$ in Eqs. (6a), (6b) and (8) can be calculated:

$$
\begin{aligned}
C_{1} & =\frac{a\left(-A_{3} k_{2} \sinh \left(k_{1} L\right) \cos \left(k_{2} L\right)+A_{4} k_{1} \cosh \left(k_{1} L\right) \sin \left(k_{2} L\right)\right)}{\left(A_{1} A_{3} k_{2}-A_{2} A_{4} k_{1}\right) \cosh \left(k_{1} L\right) \cos \left(k_{2} L\right)-\left(A_{1} A_{4} k_{1}+A_{2} A_{3} k_{2}\right) \sinh \left(k_{1} L\right) \sin \left(k_{2} L\right)-A_{1} A_{4} k_{2}+A_{2} A_{3} k_{1}}, \\
C_{2} & =C_{1} \frac{A_{3} k_{2} \cosh \left(k_{1} L\right) \cos \left(k_{2} L\right)-A_{4}\left(k_{1} \sinh \left(k_{1} L\right) \sin \left(k_{2} L\right)+k_{2}\right)}{-A_{3} k_{2} \sinh \left(k_{1} L\right) \cos \left(k_{2} L\right)+A_{4} k_{1} \cosh \left(k_{1} L\right) \sin \left(k_{2} L\right)} \\
C_{3} & =-C_{1}, \\
C_{4} & =C_{1} \frac{A_{4} k_{1} \cosh \left(k_{1} L\right) \cos \left(k_{2} L\right)+A_{3}\left(k_{2} \sinh \left(k_{1} L\right) \sin \left(k_{2} L\right)-k_{1}\right)}{-A_{3} k_{2} \sinh \left(k_{1} L\right) \cos \left(k_{2} L\right)+A_{4} k_{1} \cosh \left(k_{1} L\right) \sin \left(k_{2} L\right)} .
\end{aligned}
$$

Equations (6)-(10) allow absolute displacement amplitudes of the spring axis under kinematic excitation of amplitude $a$ to be calculated. The natural frequency for transverse vibration of the spring presented in Fig. 2 can also be obtained using the above equations. For this purpose, in the second of the boundary conditions (8), the variable $a$ placed on the right side of the equation should be replaced by 0 . Next, based on b.c. 2 and b.c. 3 , the constants $C_{2}$ and $C_{4}$ have to be determined again while taking $C_{1}=$ const., and using b.c. 4 as the angular frequency equation. After transformations, its final form is the following:

$$
\left(A_{1} A_{3} k_{2}-A_{2} A_{4} k_{1}\right) \cosh \left(k_{1} L\right) \cos \left(k_{2} L\right)-\left(A_{1} A_{4} k_{1}+A_{2} A_{3} k_{2}\right) \sinh \left(k_{1} L\right) \sin \left(k_{2} L\right)-A_{1} A_{4} k_{2}+A_{2} A_{3} k_{1}=0 .
$$

\section{Comparison of the results of the presented analytical model with the results of the FEM model}

To validate the results obtained from the analytical model, they were compared with values from FEM simulations performed in an ANSYS environment. They were conducted for four variants of spring geometry which took their real, i.e. helical, shape into account. Their characteristic dimensions are listed in Table 1 . In each case, the same material parameters were used: Young's modulus $E=207,000 \mathrm{MPa}$, density $7860 \mathrm{~kg} / \mathrm{m}^{3}$, Poisson's ratio 0.3 , and specific damping capacity $\xi=0.001$. The specific damping capacity $\xi$ is defined as the ratio of dissipated energy to stored energy per unit volume in cyclic loading. Discretization of the model was performed with a 189BEAM element based on Timoshenko beam theory.

Modal and harmonic response analysis was conducted for each of four spring geometries. The first four natural frequencies of transversal vibration $f_{I 1}, f_{I 2}$ and $f_{I I 1}, f_{I I 2}$ were determined for fixed-free boundary conditions. A comparison of results with the frequencies $f_{I}$ and $f_{I I}$ obtained from Eq. (11) is listed in 
Table 1 Geometrical parameters of the tested springs

\begin{tabular}{lllll}
\hline & Wire diameter $d(\mathrm{~m})$ & Spring diameter $D(\mathrm{~m})$ & Number of coils $n(-)$ & Pitch $h(\mathrm{~m})$ \\
\hline Spring A & 0.007 & 0.049 & 13 & 0.0182 \\
Spring B & 0.007 & 0.035 & 18.5 & 0.0128 \\
Spring C & 0.007 & 0.028 & 13.25 & 0.009 \\
Spring D & 0.005 & 0.030 & 16.75 & 0.008 \\
\hline
\end{tabular}

Table 2 Comparison of the natural frequency results

\begin{tabular}{llcccc}
\hline Spring: & Model: & $f_{I 1}(\mathrm{~Hz})$ & $f_{I 2}(\mathrm{~Hz})$ & $f_{I I 1}(\mathrm{~Hz})$ & $f_{I I 2}(\mathrm{~Hz})$ \\
\hline Spring A & Analytical & 9.618 & 9.618 & 53.649 & 53.649 \\
& FEM & 9.600 & 9.626 & 53.441 & 53.540 \\
& $\Delta_{f}[\%]$ & $-0.19 \%$ & $0.08 \%$ & $-0.39 \%$ & $-0.20 \%$ \\
Spring B & Analytical & 9.560 & 9.560 & 56.175 & 56.175 \\
& FEM & 9.546 & 9.565 & 55.905 & 56.415 \\
& $\Delta_{f}[\%]$ & $-0.15 \%$ & $0.05 \%$ & $-0.48 \%$ & $0.43 \%$ \\
Spring C & Analytical & 32.612 & 32.612 & 177.078 & 177.078 \\
& FEM & 32.511 & 32.609 & 175.370 & 175.949 \\
& $\Delta_{f}[\%]$ & $-0.31 \%$ & $-0.01 \%$ & 84.345 & $-0.64 \%$ \\
Spring D & Analytical & 15.366 & 15.366 & 83.966 & 84.345 \\
& FEM & 15.337 & 15.374 & 84.165 \\
& $\Delta_{f}[\%]$ & $-0.19 \%$ & $0.05 \%$ & $-0.45 \%$ & $-0.21 \%$ \\
\hline
\end{tabular}

Table 3 Comparison of the nodal point coordinate results

\begin{tabular}{llllll}
\hline Spring: & Model: & $z_{N}[\mathrm{~m}]\left(f=f_{1}\right)$ & $z_{N}[\mathrm{~m}]\left(f=f_{2}\right)$ & $z_{N}[\mathrm{~m}]\left(f=f_{3}\right)$ & $z_{N}[\mathrm{~m}]\left(f=f_{4}\right)$ \\
\hline Spring A & Analytical & 0.14876 & 0.16888 & 0.17731 & 0.18249 \\
& FEM & 0.14909 & 0.16941 & 0.17654 & 0.20247 \\
& $\Delta_{z}[\%]$ & $0,22 \%$ & $0,31 \%$ & $-0,44 \%$ & $9,87 \%$ \\
Spring B & Analytical & 0.149947 & 0.169073 & 0.177119 & 0.182100 \\
& FEM & 0.15019 & 0.168747 & 0.177813 & 0.180907 \\
& $\Delta_{z}[\%]$ & $0.16 \%$ & $-0.19 \%$ & $0.39 \%$ & $-0.66 \%$ \\
Spring C & Analytical & 0.074705 & 0.085120 & 0.089477 & 0.092128 \\
& FEM & 0.074775 & 0.085125 & 0.089325 & 0.09360 \\
\multirow{3}{*}{ Spring D } & $\Delta_{z}[\%]$ & $0.09 \%$ & $0.01 \%$ & $-0.17 \%$ & $1.57 \%$ \\
& Analytical & 0.084038 & 0.095640 & 0.10049 & 0.10345 \\
& FEM & 0.084067 & 0.095733 & 0.10107 & 0.10667 \\
& $\Delta_{z}[\%]$ & $0.03 \%$ & $0.10 \%$ & $0.57 \%$ & $3.02 \%$ \\
\hline
\end{tabular}

Table 2. Then, four intermediate frequencies were determined for each of the four springs, calculated according to the following relationship:

$$
f_{i}=f_{I}+\frac{f_{I I}-f_{I}}{5} \cdot i \quad i=1,2,3,4 .
$$

These 16 frequencies were subsequently utilized in harmonic response analyses, which enabled determination in each case of the position of the nodal point, defined by the coordinate $z_{N}$ and maximum amplitude $\bar{W}_{\max }$. In each of the performed analyses, the kinematic excitation amplitude was equal to $0.01 \mathrm{~m}$. A comparison of the FEM results with analytical model results is shown in Tables 3 and 4. In each case, the relative difference $\Delta$ between the FEM model and analytical model results was calculated as the difference between these results divided by the FEM model result.

The values of natural frequencies presented in Table 2 indicate very good compliance between these two modelling approaches. Differences between the values obtained with the help of FEM simulations and the analytical model in each case were lower than $1 \%$.

Further investigating the data in Tables 3 and 4, an agreement between models can be found regarding the position of the nodal point $z_{N}$ and maximum amplitude $\bar{W}_{\text {max }}$. The differences between models were higher only in two instances, especially in relation to the amplitude $\bar{W}_{\text {max }}$. The reason behind this in both cases is the proximity of excitation frequency $\left(f=f_{4}\right)$ to the first mode of torsional vibrations. This is presented in Fig. 3 which shows the amplitudes of deformations in the $x$-direction for springs $\mathrm{A}$ and $\mathrm{B}$. 
Table 4 Comparison of the maximum displacement amplitude results

\begin{tabular}{llcccc}
\hline Spring: & Model: & $\left|\bar{W}_{\text {max }}\right|[\mathrm{m}]\left(f=f_{1}\right)$ & $\left|\bar{W}_{\text {max }}\right|[\mathrm{m}]\left(f=f_{2}\right)$ & $\left|\bar{W}_{\text {max }}\right|[\mathrm{m}]\left(f=f_{3}\right)$ & $\left|\bar{W}_{\max }\right|[\mathrm{m}]\left(f=f_{4}\right)$ \\
\hline Spring A & Analytical & 0.011976 & 0.010006 & 0.012574 & 0.023608 \\
& FEM & 0.011958 & 0.010005 & 0.012608 & 0.033868 \\
& $\Delta_{W}[\%]$ & $-0.15 \%$ & $-0.01 \%$ & $0.27 \%$ & $0.29 \%$ \\
Spring B & Analytical & 0.011763 & 0.010071 & 0.012843 & 0.024346 \\
& FEM & 0.011751 & 0.010074 & 0.012888 & 0.02471 \\
& $\Delta_{W}[\%]$ & $-0.1 \%$ & $0.03 \%$ & $0.35 \%$ & $0.47 \%$ \\
Spring C & Analytical & 0.012091 & 0.009976 & 0.012438 & 0.023240 \\
& FEM & 0.012064 & 0.010102 & 0.012383 & $-3.44 \%$ \\
Spring D & $\Delta_{W}[\%]$ & $-0.22 \%$ & $1.24 \%$ & $-0.44 \%$ & 0.023375 \\
& Analytical & 0.012053 & 0.009985 & 0.012487 & 0.026496 \\
& FEM & 0.012026 & 0.00993 & 0.012303 & $11.78 \%$ \\
& $\Delta_{W}[\%]$ & $-0.22 \%$ & $-0.55 \%$ & $-1.496 \%$ & \\
\hline
\end{tabular}

(a)

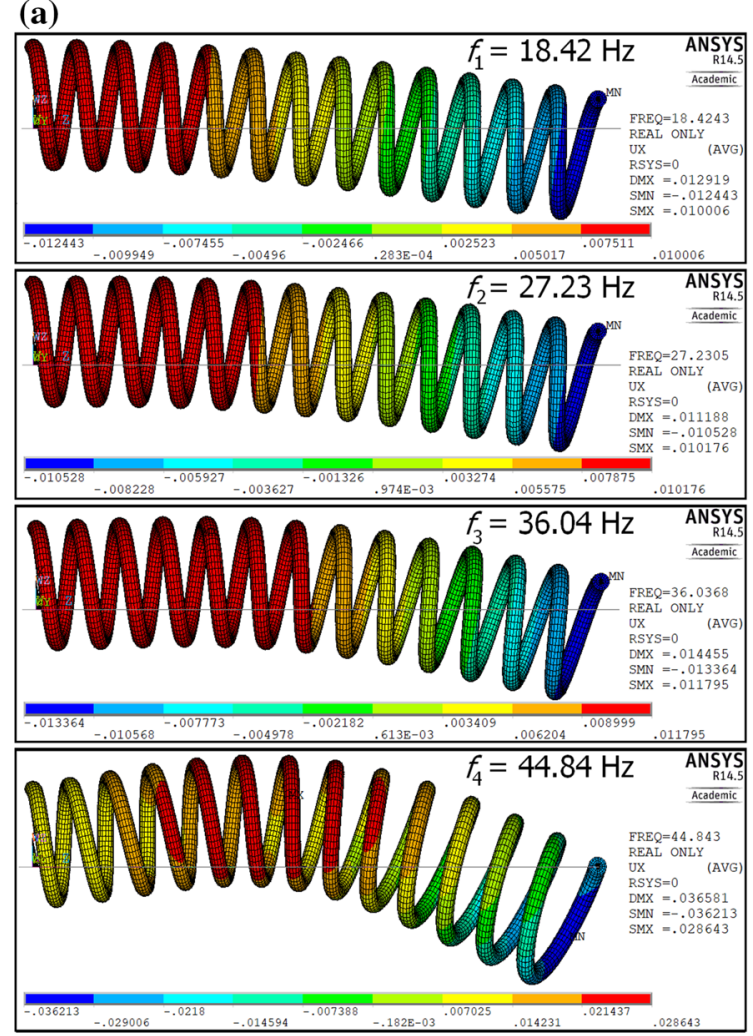

(b)

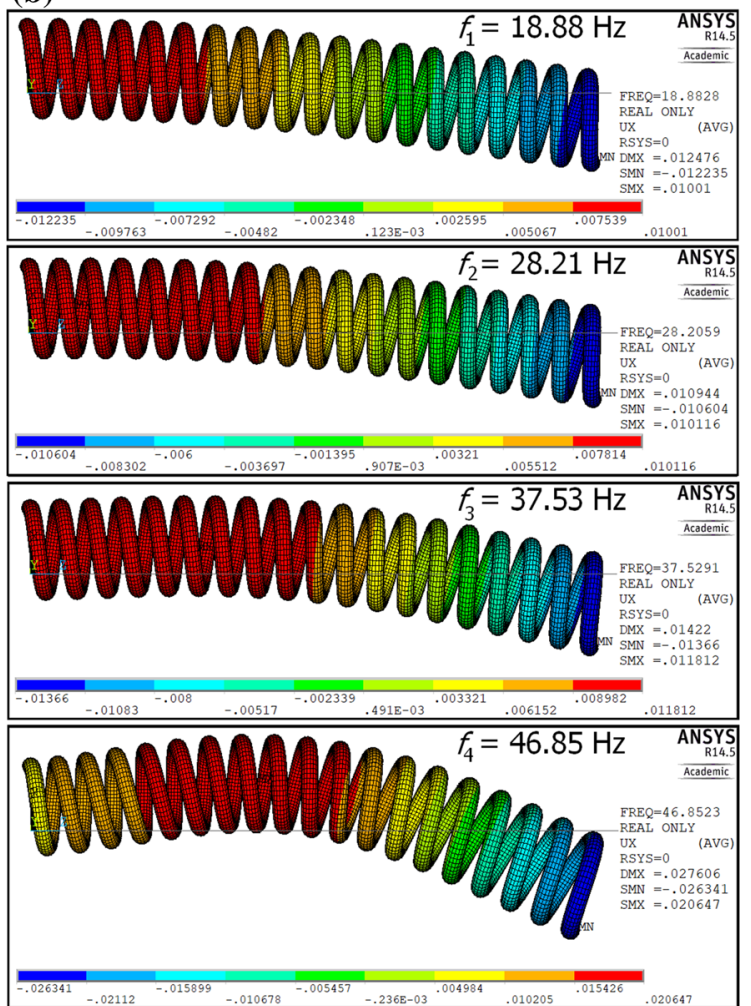

Fig. 3 Real-only component of displacement amplitudes of FE models under excitation frequencies $f_{1}, f_{2}, f_{3}$, and $f_{4}$ : spring A a, spring B b

As can be seen in the case of vibration of spring A, for the excitation frequency $f_{4}=44.84 \mathrm{~Hz}$ the form of the transversal bending mode is accompanied by the torsional mode. This is because the calculated first torsional frequency is equal to $45.34 \mathrm{~Hz}$. A similar situation, however to a lesser degree, also occurs in the case of spring D. The obtained results show that the analytical model should not be used in the described conditions. This inconvenience can be easily eliminated if the geometric parameters of the spring are selected in such a way that ensures movement of the torsional frequency away from the first bending mode frequency. The first torsional frequency for the given fixed-free boundary conditions can be obtained using the analogous formula for circular shafts, which can be found, for example, in [19]. This formula can be written as

$$
f_{\text {Itor }}=\frac{1}{4} \sqrt{\frac{\chi}{I}},
$$




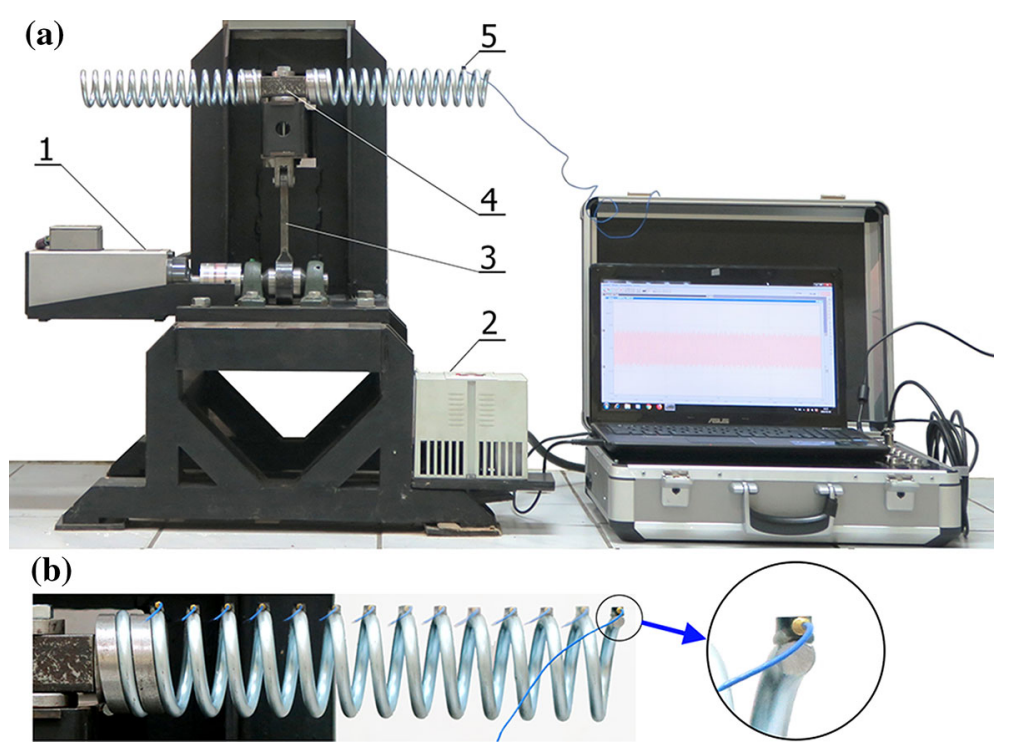

Fig. 4 Experimental stand: a rigid frame with kinematic eccentric exciter and measurement apparatus, $\mathbf{b}$ locations of accelerometer attachment on the spring

where $\chi$ represents the torsional stiffness of the shaft and $I$ represents its rotational inertia. The equivalent rotational inertia of the spring equals $m_{u} L R^{2}$, while the torsional stiffness of the helical spring can be expressed as [20]

$$
\chi=\frac{E J \cos \delta}{\pi \mathrm{D} n\left((1+v) \sin ^{2} \delta+\cos ^{2} \delta\right)} .
$$

By substituting the proper value of the rotational inertia and the torsional stiffness in Eq. (12), the first natural frequency of torsional vibrations of the spring can be expressed as

$$
f_{\text {Itor }}=\frac{1}{2} \sqrt{\frac{E J \cos \delta}{\pi D^{3} n\left((1+v) \sin ^{2} \delta+\cos ^{2} \delta\right) m_{u} L}} .
$$

\section{Experimental tests}

The test rig on which the experimental tests verifying the analytical model in terms of nodal point position were conducted is presented in Fig. 4a. The electric motor $\mathbf{1}$ mounted to the rig frame and driven by the variable-frequency drive $\mathbf{2}$ is connected by means of an elastic coupling with an eccentric shaft, of $0.2 \mathrm{~mm}$ eccentricity. The eccentric shaft cooperates with the connecting rod $\mathbf{3}$ with a head connected to the spring holder 4, which in turn is mounted to the frame by means of elastic slats in such a way that the holder can only move in a vertical direction. The investigated springs were mounted to the spring holder, which vibrated at a fixed frequency and amplitude of $0.2 \mathrm{~mm}$. The experimental research consisted of measuring the time course of the spring's vibration velocity, using a 352C22 PCB Piezotronics micro accelerometer 5, as shown in Fig. 4a. The investigated springs had parameters in accordance with those of spring A, as presented in Table 1.

Experimental tests were performed for four different excitation frequencies located between $f_{I}$ and $f_{I I}$ : $11.06 \mathrm{~Hz}, 23.15 \mathrm{~Hz}, 35.60 \mathrm{~Hz}$, and $47.79 \mathrm{~Hz}$. Measurement data were registered with a SENSOR KSD400 vibration analyser. The sampling frequency was $8192 \mathrm{~Hz}$ and measurement duration $4 \mathrm{~s}$. Based on the spring's steady response due to excitation, the RMS values of vibration velocity amplitude were registered at 14 consecutive points of the spring, as shown in Fig. $4 \mathrm{~b}$.

The RMS values of the measured vibration velocity amplitude with consideration of the sign change due to zero crossing for each of four excitation frequencies are shown as dots in Fig. 5. Then, a nonlinear curve fitting procedure was carried out in the MAPLE computing environment, using a fitting function of the same form as the amplitude Eq. (6). 


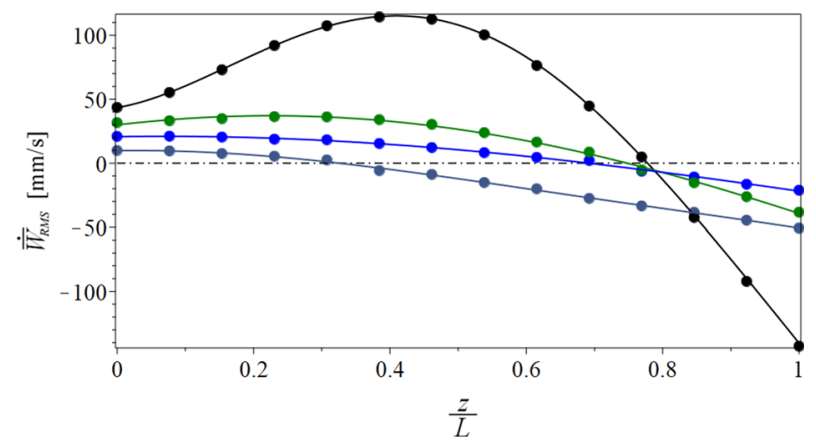

Fig. 5 Results of experimental measurements of RMS values of vibration velocity (dots) together with the approximation curves for four different excitation frequencies

Table 5 Comparison of the nodal point positions obtained from the analytical model and from experiments

\begin{tabular}{lcccc}
\hline Frequency $[\mathrm{Hz}]$ & 11.06 & 23.15 & 35.60 & 47.79 \\
\hline$z_{N} / L($ analytical $)$ & 0.3268 & 0.6855 & 0.7481 & 0.7773 \\
$z_{N} / L($ experimental $)$ & 0.3251 & 0.6909 & 0.7475 & 0.7777 \\
$\Delta_{Z N}[\%]$ & $-0.5 \%$ & $0.8 \%$ & $-0.1 \%$ & $0.05 \%$ \\
\hline
\end{tabular}

Utilizing the obtained approximation functions, which are also presented in Fig. 5, their roots were computed, giving in the same way the positions of nodal points. The nodal point coordinates $z_{N}$ obtained on the basis of an analytical model and experiments are compared in Table 5.

The presented experimental results confirm the high accuracy of the developed analytical model. However, it should be noted that the maximum amplitudes of the spring axis deflection were relatively small. The maximum amplitude of deflection occurred at the forcing frequency of $11.06 \mathrm{~Hz}$ and exceeded the value of $1 \mathrm{~mm}$, and therefore the quotient $\bar{W}_{\max } / L$ was approx. 0.0043 .

\section{Analysis of the nodal point position}

The theoretical model allows the influence of excitation frequency on the location of the nodal point and amplitude value of displacement and angle along the spring axis to be estimated. Plots in Fig. 6 show displacement amplitudes for a spring with the same geometric parameters as spring $\mathrm{A}$ in Table 1, undergoing the kinematic excitation of amplitude $a=0.005 \mathrm{~m}$. In total, responses for the ten excitation frequencies with the step of $4.0028 \mathrm{~Hz}$ between the first and the second natural transverse vibration frequencies were drawn. For a given spring of type $\mathrm{A}$, these frequencies are accordingly $f_{I}=9.618 \mathrm{~Hz}$ and $f_{I I}=53.649 \mathrm{~Hz}$. Numerical calculations were conducted using the MAPLE computing environment.

Investigating the plots of amplitudes presented in Fig. 6, it can be observed that the position of nodal points (red dots) moves in the direction of the free-end of the spring with increasing excitation frequency. The closer the excitation frequency to the second natural frequency $f_{I I}$, the lesser the gain of distance $z_{N}$. An increase in $z_{N}$ is accompanied by a decrease in static stiffness of the joint between the vibrating object and vibroisolated base. This-for relatively high values of $z_{N}$-leads to a significant increase in displacement amplitude along the spring, which is an undesirable outcome. However, when for given system parameters, excitation frequency is only slightly bigger than the first resonance frequency, the obtained static stiffness is very high. At the same time, displacement amplitudes of the spring axis and deflection angle amplitude at the nodal point reach very high values, which is undesirable as the isolated object would be mounted in this place.

The transverse stiffness of a helical spring, working in nodalised beam conditions, can be evaluated based on the equivalent beam concept. Static deflection of the spring axis $u_{N}$ at the nodal point $N$, where a transverse force $Q$ is applied, can be calculated as a sum of deflection caused by bending and shearing of the equivalent beam. Using known relationships [21], this deflection can be written in the form

$$
u_{N}=Q z_{N}\left(\frac{z_{N}^{2}}{3 \alpha}+\frac{1}{\beta}\right) \text {. }
$$




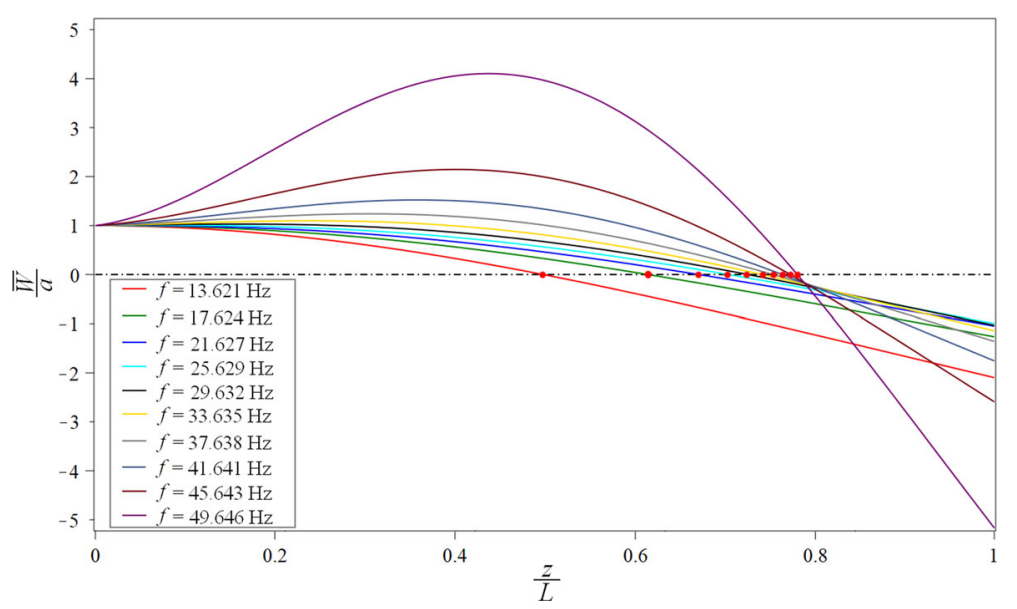

Fig. 6 Vibrating shape response of spring A for selected excitation frequencies
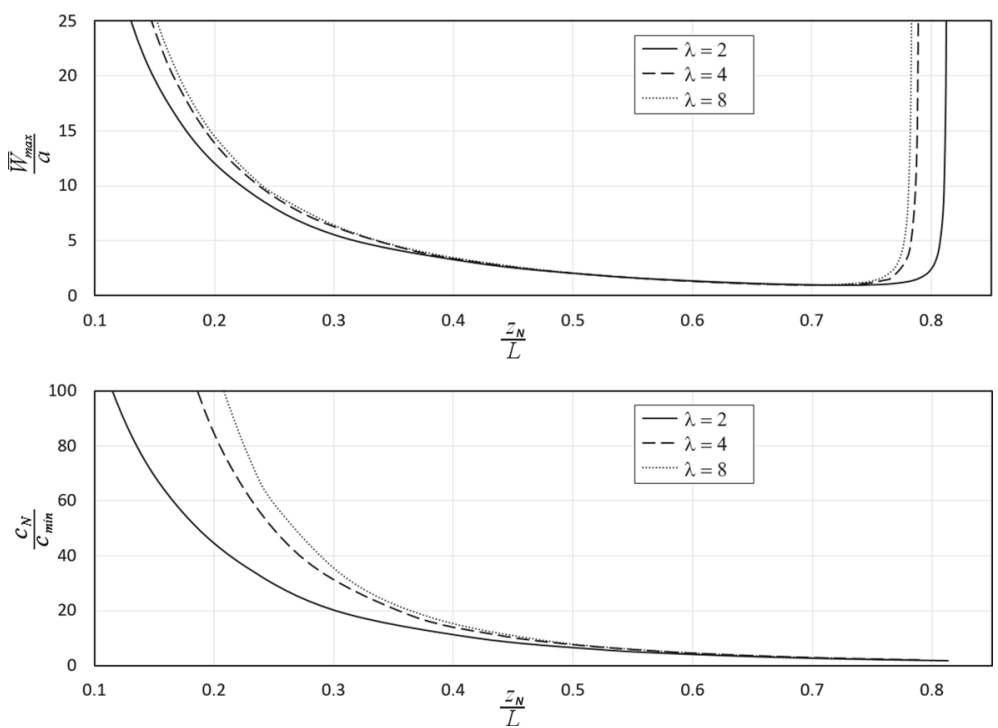

Fig. 7 a The maximum relative spring deflection amplitude and $\mathbf{b}$ relative transverse stiffness of the spring as a function of nodal point position for three different values of spring slenderness

Therefore, the static transverse stiffness of a spring in a nodal point can be expressed as:

$$
c_{N}=\frac{3 \alpha \beta}{z_{N}\left(z_{N}^{2} \beta+3 \alpha\right)} \text {. }
$$

It should be noted that the maximum value of $z_{N}$ for $f \rightarrow f_{I I}$ increases with a reduction in the spring slenderness $\lambda$, described as the ratio between the spring length $L$ and its nominal diameter $D$. Additionally, the relation between the nodal point position $z_{N}$ and the maximum spring deflection amplitude $\bar{W}_{\text {max }}$ depends on the spring slenderness $\lambda$. These relations are analysed in Fig. 7, which was developed based on the analytical model presented. In Fig. 7a, the relation between the quotient of maximum spring deflection amplitude $\bar{W}_{\max }$ to the excitation amplitude $a$ and the quotient of nodal point position $z_{N}$ to the spring length $L$ is shown for 3 different values of spring slenderness. In Fig. 7b, the analogous relations between the quotient of transverse spring stiffness $c_{N}$ to the minimum spring stiffness $c_{\min }=c_{N}\left(z_{N}=L\right)$ and the quotient of nodal point position $z_{N}$ to the spring length $L$ are shown.

As shown in Fig. 7b, the increase in lateral stiffness accompanying a decrease in the $z_{N}$ value is more significant when the spring is more slender. It can be seen that the influence of spring slenderness on this relation is very significant. This is related to the decreasing share of shear stiffness in the transverse stiffness 
with increasing spring slenderness. The influence of slenderness on the increase in the maximum amplitude of spring deflection with a decreasing $z_{N}$ value is, in turn, much smaller, as can be noticed in Fig. $7 \mathrm{a}$.

\section{Conclusions}

The computational model presented in this paper enables an effective analysis to be conducted of a vibration isolation system using a coil spring as an elastic beam, and thus the appropriate selection of system parameters for the given operating conditions. The results of the presented model show high agreement with the results of numerical and experimental investigations. It was shown that the slenderness of the spring has a significant effect on the relative deflection amplitudes at small distances of the nodal point from the spring mounting. In contrast, in the range of the $z_{N} / L$ ratio from 0.4 to 0.75 , this effect is negligibly small.

When designing a vibration isolation system, the nodal point's position on the spring axis should be determined. In the case of small values of the ratio $Z_{N} / L$, a significant static lateral stiffness of the system is obtained. However, it is accompanied by large values of the relative deflection amplitudes. For example, for $Z_{N} / L=0.3$, the quotient of the maximum amplitude of deflection to the amplitude of excitation exceeds the value of 5. At the same time, even a small change in the actual value of the frequency of excitation in relation to the assumed value causes a relatively large change in the theoretical position of the nodal point and thus reduces the effectiveness of isolation. In the case of such a selection of the system's parameters that the $Z_{N} / L$ ratio is within limits from 0.65 to 0.75 , the lowest values of the maximum amplitudes are obtained. Simultaneously, even significant deviations of the value of the excitation frequency from the assumed value do not cause large changes in the position of the theoretical nodal point and thus do not significantly affect the effectiveness of the system. The conducted numerical analyses, in which the spring was modelled in a way reflecting its real geometry, indicated the risk of the occurrence of coupling between the transverse and the torsional form of vibration. Using relation (14), as presented in this paper, it is possible to calculate the first natural frequency of torsional vibrations of the spring and thus to design the system in such a way as to avoid the described phenomenon.

Funding This work was supported by the AGH University of Science and Technology within the scope of the research program No. 16.16.130.942.

Availability of data and material Not applicable.

Code availability Not applicable.

\section{Declaration}

Conflict of interest There are no potential conflicts of interest involved in the present study.

Open Access This article is licensed under a Creative Commons Attribution 4.0 International License, which permits use, sharing, adaptation, distribution and reproduction in any medium or format, as long as you give appropriate credit to the original author(s) and the source, provide a link to the Creative Commons licence, and indicate if changes were made. The images or other third party material in this article are included in the article's Creative Commons licence, unless indicated otherwise in a credit line to the material. If material is not included in the article's Creative Commons licence and your intended use is not permitted by statutory regulation or exceeds the permitted use, you will need to obtain permission directly from the copyright holder. To view a copy of this licence, visit http://creativecommons.org/licenses/by/4.0/.

\section{References}

1. Halwes, DR.: Nodal beam inertial flexure. U.S. Patent No 3,858,831, (1975)

2. Gaffey, T.M., Balke, R.W.: Isolation of rotor induced vibration with the bell focal pylon-nodal beam system. No. 760892 . SAE Technical Paper (1976)

3. Hao, K.Y., Ripin, Z.M.: Nodal control of grass trimmer handle vibration. Int. J. Ind. Ergon. 43(1), 18-30 (2013)

4. Tewari, V.K., Dewangan, K.N.: Effect of vibration isolators in reduction of work stress during field operation of hand tractor. Biosys. Eng. 103(2), 146-158 (2009)

5. Golycheva, E.V., Babitsky, V.I., Veprik, A.M.: Dynamic correction of excitation in hand-held electro-pneumatic percussion machines. J. Sound Vib. 259(4), 829-843 (2003) 
6. Sam, B., Kathrivel, K.: Development and evaluation of vibration isolators for reducing hand transmitted vibration of walking and riding type power tillers. Biosys. Eng. 103(4), 427-437 (2009)

7. Yeh, C., Lu, S., Lin, K., Lee, C.: An Investigation of Vibration Exposure for Use of Brushcutter, Proceedings 19th Triennial Congress of the IEA, 2015, Melbourne.

8. Xu, X.S., Dong, R.G., Welcome, D.E., Warren, C., McDowell, T.W., Wu, J.Z.: Vibrations transmitted from human hands to upper arm, shoulder, back, neck, and head. Int. J. Ind. Ergon. 62, 1-12 (2016)

9. Palmer, K.T., Bovenzi, M.: Rheumatic effects of vibration at work, Best Practice \& Research. Clin. Rheumatol. 29(3), 424-439 (2015)

10. Mahbub, M.H., Kurozawa, Y., Ishitake, T., Kume, Y., Miyashita, K., Sakakibara, H., Sato, S., Toibana, N., Harada, N.: A systematic review of diagnostic performance of quantitative tests to assess musculoskeletal disorders in hand-arm vibration syndrome. Ind. Health 53(5), 391-397 (2015)

11. Gawkrodger, D.J., Mili, S.: "Air Hammer Operators" in Kanerva's Occupational Dermatology. Springer, Berlin (2018)

12. Lai, S., Chui, J., Tong, L., Sun, J.: A human-based study of hand-arm vibration exposure limits for construction workers. J. Vib. Eng. Technol. 7(4), 379-388 (2019)

13. Majkut, L., Michalczyk, J.: "Nodalised beam" method for vibroinsulation of manually operated tools. Arch. Mech. Eng. 49(3), 215-229 (2002)

14. Michalczyk, K.: Analysis of lateral vibrations of the axially loaded helical spring. J. Theor. Appl. Mech. 53(3), 754-755 (2015)

15. Pearson, D.: The transfer matrix method for the vibration of compressed helical springs. J. Mech. Eng. Sci. 24, 163-171 (1982)

16. Lee, J., Thompson, D.J.: Dynamic stiffness formulation, free vibration and wave motion of helical springs. J. Sound Vib. 239, 297-320 (2001)

17. Li, X.Y., Zhao, X., Li, Y.H.: Green's functions of the forced vibration of Timoshenko beams with damping effect. J. Sound Vib. 333, 1781-1795 (2014)

18. Krużelecki, J., Życzkowski, M.: On the concept of an equivalent column in the stability problem of compressed helical springs. Ingenieur-Archiv 60(6), 367-377 (1990)

19. Shabana, A.A.: Vibration of Discrete and Continuous Systems, 3rd edn. Springer, New York (2019)

20. Branowski B.: Sprężyny metalowe. In: Polish, (Metal Springs), Wydawnictwo Naukowe PWN, Warsaw (1997)

21. Kobelev, V.: Durability of springs. Springer, Wien (2018)

Publisher's Note Springer Nature remains neutral with regard to jurisdictional claims in published maps and institutional affiliations. 